\title{
Does viscosity describe the kinetic barrier for crystal growth from the liquidus to the glass transition?
}

\author{
Marcio Luis Ferreira Nascimento ${ }^{1, a)}$ and Edgar Dutra Zanotto ${ }^{2, b)}$ \\ ${ }^{1}$ Institute of Humanities, Arts and Sciences, Federal University of Bahia, Rua Barão de Jeremoabo s/n, \\ Glauber Rocha Pavilion (PAF3), Ondina University Campus, 40170-115 Salvador, Bahia, Brazil \\ ${ }^{2}$ Department of Materials Engineering, Vitreous Materials Laboratory, Federal University of São Carlos, \\ 13565-905 São Carlos, São Paulo, Brazil
}

(Received 19 May 2010; accepted 30 August 2010; published online 1 November 2010)

An analysis of the kinetic coefficient of crystal growth $U_{\text {kin }}$, recently proposed by Ediger et al. [J. Chem. Phys. 128, 034709 (2008)], indicates that the Stokes-Einstein/Eyring (SE/E) equation does not describe the diffusion process controlling crystal growth rates in fragile glass-forming liquids. $U_{\text {kin }}$ was defined using the normal growth model and tested for crystal data for inorganic and organic liquids covering a viscosity range of about $10^{4}-10^{12} \mathrm{~Pa}$. Here, we revisit their interesting finding considering two other models: the screw dislocation $(S D)$ and the two-dimensional surface nucleated (2D) growth models for nine undercooled oxide liquids, in a wider temperature range, from slightly below the melting point down to the glass transition region $T_{g}$, thus covering a wider viscosity range: $10^{1}-10^{13} \mathrm{~Pa}$ s. We then propose and use normalized kinetic coefficients $\left(M_{\text {kin }}\right)$ for the SD and 2D growth models. These new kinetic coefficients restore the ability of viscosity to describe the transport part of crystal growth rates $\left(M_{\text {kin }} \sim 1 / \eta\right.$ and $\left.\xi \sim 1\right)$ from low to moderate viscosities $\left(\eta<10^{6} \mathrm{~Pa} \mathrm{~s}\right)$, and thus the SE/E equation works well in this viscosity range for all systems tested. For strong glasses, the SE/E equation works well from low to high viscosities, from the melting point down to $T_{g}$ ! However, for at least three fragile liquids, diopside (kink at $1.08 T_{g}$, $\eta=1.6 \times 10^{8} \mathrm{~Pa} \mathrm{~s}$ ), lead metasilicate (kink at $1.14 T_{g}, \eta=4.3 \times 10^{6} \mathrm{~Pa} \mathrm{~s}$ ), and lithium disilicate (kink at $1.11 T_{g}, \eta=1.6 \times 10^{8} \mathrm{~Pa} \mathrm{~s}$ ), there are clear signs of a breakdown of the SE/E equation at these higher viscosities. Our results corroborate the findings of Ediger et al. and demonstrate that viscosity data cannot be used to describe the transport part of the crystal growth (via the SE/E equation) in fragile glasses in the neighborhood of $T_{g}$. () 2010 American Institute of Physics. [doi:10.1063/1.3490793]

\section{INTRODUCTION}

The mechanisms, thermodynamics, and kinetic aspects of crystal growth in glass-forming liquids are some of the most important features for understanding and controlling the vitrification process, as well as for the development of new glass ceramics (polycrystalline materials made by controlled crystallization of special glass compositions) with usual or improved properties. The theoretical and the experimental analyses of crystal nucleation and growth kinetics in glasses and undercooled liquids and the applicability of viscosity to describe the diffusion process associated with these two phenomena have been discussed for many years and, particularly, intensively in the past two decades. ${ }^{1-3}$

The growth velocity of a crystal in an undercooled liquid $U$ can be regarded as the product of two quantities: (i) the probability that the newly captured molecules are irreversibly retained into the crystal, which can be expressed in terms of the free energy difference between the two phases, and (ii) the rate at which molecules of the liquid adjacent to

\footnotetext{
${ }^{\text {a) }}$ Also at PROTEC/PEI-Postgraduate Program in Industrial Engineering, Polytechnic School, Federal University of Bahia, Rua Aristides Novis 2, Federação, 40210-630 Salvador, Bahia, Brazil. URL: www.protec.ufba.br. Electronic mail:mlfn@ufba.br.

${ }^{b)}$ Electronic mail: dedz@ufscar.br.
}

the growth front can diffuse and organize into a crystal (the kinetic coefficient $U_{\text {kin }}$ ), i.e., the attachment process that reflects the dynamics of collective fluctuations at the crystalliquid interface.

A classical crystal growth model, denoted as continuous or normal growth model, was derived by Wilson ${ }^{2}$ and Frenkel, ${ }^{3}$ in which $U_{\text {kin }}$ is associated with an activated process for atoms or molecules to move from the liquid to a growing crystal. A similar growth model is the screw dislocation, ${ }^{4}$ but in this model atoms are preferentially attached to screw dislocations on the surface of the growing crystal. For both cases, the transport process is assumed to be similar to that which governs self-diffusion. At small undercoolings, just below the melting point, the temperature dependence of the diffusion coefficient $D$ is often assumed to be described by the inverse shear viscosity $\eta^{-1}$, i.e., by the Stokes-Einstein/Eyring (SE/E) equation. Such assumption is quite handy because viscosity is the most readily available transport coefficient in highly viscous liquids. Using this approximation, these two growth models have met with success over the modest and intermediate undercoolings, starting from the melting point $T_{m}$ down to $\approx 1.1 T_{g}$ for two inorganic glass-forming liquids: diopside ${ }^{4}$ and silica. ${ }^{5}$ On the other hand, Swallen et al. ${ }^{6,7}$ suggested that below $\approx 1.2 T_{g}, D$ and $\eta$ have different temperature dependences for an organic liq- 
uid, tris- $\alpha$-naphthylbenzene. Similar results have been observed from Mapes et $a l^{8}{ }^{8}$ for $o$-terphenyl. However, to the best of our knowledge, there is no firm proof of the breakdown of the SE/E equation at a sufficiently low temperature for inorganic glass-forming liquids.

An analysis of the kinetic coefficient of crystal growth, $U_{\text {kin }}(T)=U(T) /\left[1-\exp \left(-\Delta G(T) / k_{B} T\right)\right]$, where $U$ is the crystal growth rate, $\Delta G$ is the thermodynamic driving force, $T$ is the temperature, and $k_{B}$ is the Boltzmann constant recently proposed by Ediger et al., ${ }^{9}$ indicates that the StokesEinstein/Eyring equation does not describe the transport controlling crystal growth rates in fragile glass-forming liquids. Ediger et al. demonstrated that $U_{\text {kin }}$ scales with $\eta^{-\xi}$, and the exponent $\xi$ depends systematically on the fragility of the liquid: the greater the fragility, the lower the value of $\xi$. If the $\mathrm{SE} / \mathrm{E}$ equation was valid, $\xi=1$, a value that was found only for the strongest liquids, such as $\mathrm{SiO}_{2}$ and $\mathrm{GeO}_{2}$. In Ref. 9, $U_{\text {kin }}$ was defined using the normal growth model and tested for crystal data for inorganic and organic liquids covering a viscosity range of about $10^{4}-10^{12} \mathrm{~Pa} \mathrm{~s}$. For the organic liquids, all of the data analyzed were below $1.25 T_{g}$ (the typical decoupling temperature); for the oxide glasses, the data included somewhat higher temperatures. Thus, their finding that the SE/E equation does not describe the transport part of the crystal growth corroborates the suggestions of other authors; the new finding is that the departure from the predicted behavior was more intense for more fragile liquids.

In this article, we focus on nine stoichiometric oxide glass-forming systems with widely different fragilities (20 $<m<60$ ) that crystallize without obvious compositional changes, i.e., they undergo polymorphic crystallization. The used data span a very wide range of viscosities from $10^{1}$ to $10^{13} \mathrm{~Pa}$ s. We follow the approach of Ediger et al. ${ }^{9}$ to calculate $U_{\text {kin }}$, but we compare the transport part of the crystal growth $U_{\text {kin }}$ with $\eta^{-1}$ using two other growth models instead of the normal growth model. Additionally, we introduce two modified forms of the kinetic coefficient to fully test the possible influence of the growth models and their preexponential factors, as described below. In summary, our objective is to test whether (i) viscosity describes the transport part controlling the crystal growth all the way from $T_{m}$ to $T_{g}$; (ii) viscosity can describe only a part of the growth curve, with a breakdown at $1.1 T_{g}-1.2 T_{g}$; or (iii) it fails at all temperatures below the melting point. For clarity, these three possibilities are schematically shown in Fig. 1.

\section{SUMMARY OF CRYSTAL GROWTH RATE MODELS}

In discussing the crystal growth from the melt, it should be recognized that the nature of the crystal/melt interface plays a decisive influence on crystallization kinetics. As we shall see, each one of the standard growth models is based on a particular assumption concerning the relative amount and the nature of interfacial sites where atoms can be added or removed. Three standard models are often used to describe the crystal growth process in inorganic glasses, which are based on different views of the nature of the crystal/liquid interface. These models are (i) the screw dislocation model, (ii) the normal or continuous growth model, and (iii) the

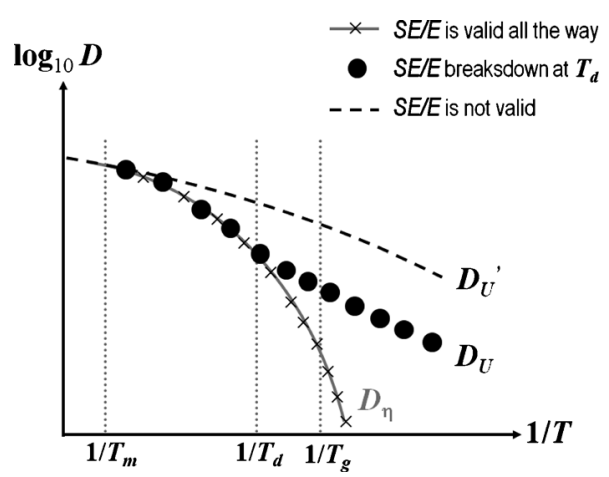

FIG. 1. Schematic representation of three possibilities for the SE/E equation: (i) full line, gray: $D_{\eta}$ calculated via viscosity by the $\mathrm{SE} / \mathrm{E}$ equation; $D_{U}$ (×) agrees with $D_{\eta}$ all the way; (ii) $D_{U}(\bullet)$ breaks down at $T_{d}>T_{g}$; and (iii) $D_{U}^{\prime} \neq D_{\eta}$, i.e., the two diffusivities are different.

two-dimensional secondary surface nucleation growth model. All these models are based on the assumption that the release of latent heat does not substantially alter the crystalmelt interface temperature and that only interfacial rearrangements occur at the interface (no long range diffusion is necessary). Such models are summarized in the following paragraphs.

\section{A. The screw dislocation growth model}

The screw dislocation growth model views the interface as smooth but imperfect on an atomic scale, with growth taking place at step sites provided by screw dislocations intersecting the interface. The crystal growth rate $U$ is given by ${ }^{10,11}$

$$
U=f \frac{D}{\lambda}\left[1-\exp \left(-\frac{\Delta G}{k_{B} T}\right)\right]=f \frac{k_{B} T}{\lambda^{2} \eta}\left[1-\exp \left(-\frac{\Delta G}{k_{B} T}\right)\right],
$$

where $\eta$ is the shear viscosity, $\lambda$ is the distance advanced by the interface in a unit kinetic process (usually taken as a molecular diameter), $\Delta G$ is the thermodynamic driving force for crystallization, i.e., the difference between the free energies of the undercooled melt and crystalline phase, $T$ is the absolute temperature, and $k_{B}$ is the Boltzmann constant.

In Eq. (1), $D$ is the diffusion coefficient controlling the molecular rearrangements at the crystal/liquid interface and is often related to the viscosity by the Stokes-Einstein/ Eyring equation: $D=k_{B} T / \lambda \eta$. For one-component systems, one could expect good agreement between certain crystal growth models and experiment when employing viscosity to calculate the transport part, at least from the melting point down to $T_{d} \sim 1.1 T_{g}-1.2 T_{g}$, i.e., $D_{U}$ (Refs. 4 and 5) is quite likely proportional to $1 / \eta$ at temperatures above $T_{d}$. However, for multicomponent systems, a description of diffusion in terms of viscosity becomes questionable even at higher temperatures, ${ }^{1}$ and the actual diffusion coefficient controlling crystal growth must be replaced by an effective diffusion coefficient $D_{\text {eff }}$, which is a combination of the diffusion coefficients of all components. These hypotheses will be tested here. 
The fraction of sites on the interface where atoms of the liquid can preferentially be added to or removed from the crystal $f$ is given by

$$
f=\frac{\lambda \Delta G}{4 \pi \gamma V_{m}},
$$

where $\gamma$ is the specific surface energy of the liquid/crystal interface and $V_{m}$ is the molar volume of the crystal. According to Ref. 10, it is possible to define $\gamma$ as

$$
\gamma=\alpha \Delta H_{m} / \sqrt[3]{V_{m}^{2} N_{A}},
$$

where $N_{A}$ is the Avogadro's number and $\Delta H_{m}$ is the melting enthalpy. The fraction of the preferred site $f$ can then be calculated by Eq. (2) using the empirical coefficient $\alpha(0.3-$ 0.5 ) in Eq. (3). Thus, consider $\alpha=0.5, f \approx \Delta T / 2 \pi T_{m}$, where $T_{m}$ is the thermodynamic melting point and $\Delta T=T_{m}-T$ is the undercooling.

When experimental data are not available, the free energy barrier $\Delta G$ can be estimated in two ways: by the Thomson/Turnbull [Eq. (4a)] or Hoffman [Eq. (4b)] approximations. These give upper and lower bounds to experimental $\Delta G$, respectively,

$$
\begin{aligned}
& \Delta G=\Delta H_{m} \frac{\Delta T}{T_{m}} \quad \text { (Thomson/Turnbull), } \\
& \Delta G=\Delta H_{m} \frac{\Delta T \cdot T}{T_{m}^{2}} \quad \text { (Hoffman). }
\end{aligned}
$$

A previous analysis ${ }^{4}$ of these two equations has shown that they yield almost identical results at high temperatures for some glasses. In this work, for brevity, we use only Thomson's approximation [Eq. (4a)].

\section{B. Normal growth model}

According to the normal growth model, atoms can be added to or removed from any site on the crystal-liquid interface, and the individual molecular process in crystallization is treated as simply activated. Therefore, such interface is pictured as rough on an atomic scale and all the sites on the interface are assumed to be equivalent growth sites $(f$ $\approx 1$ ). The growth rate is expressed by Eq. (1) with $f=1$. Note that for this and the previous model, the only one unknown parameter is the jump distance $\lambda$. All other parameters are known or can be estimated.

\section{Two-dimensional surface nucleation growth}

From the two-dimensional (2D) growth model, the surface is considered atomically smooth and free from defects. Growth occurs by the formation of two-dimensional nuclei on top of the primary crystals, which grow laterally. The growth rate is expressed by ${ }^{10,11}$

$$
U=C \frac{D}{\lambda^{2}} \exp \left(-\frac{Z}{T \Delta G}\right)=C \frac{k_{B} T}{\lambda^{3} \eta} \exp \left(-\frac{Z}{T \Delta G}\right) .
$$

In the above equation, $Z$ is given by one of the following expressions, depending on the size of the secondary crystal relative to the size of the primary crystal:

$$
\begin{aligned}
& Z=\frac{\pi \lambda V_{m} \gamma^{2}}{k_{B}} \quad \text { (small crystal), } \\
& Z=\frac{\pi \lambda V_{m} \gamma^{2}}{3 k_{B}} \quad \text { (large crystal), }
\end{aligned}
$$

where $\gamma$ is the surface edge energy of the 2D crystal for growth, usually taken as the liquid-crystal surface energy cited above.

The terms small and large crystal in Eqs. (6a) and (6b), respectively, refer to two general cases: small crystal is valid when the base crystals are much larger than the 2D crystals growing on their surfaces, while large crystal applies when the secondary $2 \mathrm{D}$ crystals have sizes similar to the primary ones. ${ }^{10}$ Or when the secondary nuclei grow at a time shorter than the time period between nucleation events, one has a "small" crystal case. In the opposite situation, one has a "large" crystal case.

One should notice that these two cases are related by a factor of 3 in $Z$. In Eq. (5), $C$ is given by

$$
\begin{aligned}
& C=\lambda N_{S} A_{0} \quad(\text { small crystal), } \\
& C=\frac{\sqrt[3]{\pi N_{S} \lambda^{5} / 3}}{\Gamma(4 / 3)}\left[1-\exp \left(-\Delta G / k_{B} T\right)\right]^{2 / 3} \quad \text { (large crystal), }
\end{aligned}
$$

where $A_{0}$ is the interface area of the growing crystal, $N_{S}$ is the number of molecules (f.u.) per unit area of interface, and $\Gamma$ is the gamma function. From Eq. (7b), it is possible to note that $C=C(T)$ is a function that is weakly temperature dependent. In this work, we will consider only the large crystal case and $N_{S} \approx \sqrt[3]{N_{V}^{2}}$, where $N_{V} \approx 10^{28}$ f.u. $/ \mathrm{m}^{3}$, and the surface energy $\gamma$ as free parameters. For this particular model, we will fix the jump distance $\lambda=1 \AA$.

Regarding the general trends about crystal growth processes, Jackson et al. ${ }^{11}$ showed that the operative growth mechanism can be inferred from a plot of the reduced growth rate $U_{R}$ [Eq. (8a)] versus undercooling $\Delta T$,

$$
U_{R}=\frac{U \eta}{1-\exp \left(-\Delta G / k_{B} T\right)}=f \frac{k_{B} T}{\lambda^{2}},
$$

where $U_{R}$ gives information on the temperature dependence on the fraction of the preferred growth sites $f$ at the interface. The proposal of Ediger et al. ${ }^{9}$ is based on this equation.

Please note that Eq. (8a) refers to the normal and screw dislocation growth models, choosing appropriate $f$ values. Considering the same approach, for the 2D crystal growth case, the expression for the reduced growth rate should be

$$
U_{R}=U \eta \exp \left(\frac{Z}{T \Delta G}\right)=C \frac{k_{B} T}{\lambda^{3}} \exp \left(\frac{Z}{T \Delta G}\right) .
$$

Ediger et al. ${ }^{9}$ defined $U_{\text {kin }}$ in terms of the observed growth rate $U$ from Eqs. (8a) and (8b), 


$$
U_{\mathrm{kin}}^{N \mathrm{SD}}=\frac{U}{1-\exp \left(-\Delta G / k_{B} T\right)}=f \frac{D}{\lambda}=f \frac{k_{B} T}{\lambda^{2} \eta},
$$

where the superscripts $N$ and SD refer to the application of the normal and screw dislocation mechanisms, respectively [i.e., using $f=1$ or $f=f(T)$ ].

From the same reasons considered to define $U_{R}$ [Eqs. (8a) and (8b)], it is possible to define a different $U_{\text {kin }}$ term for the $2 \mathrm{D}$ growth mechanism, which was not considered by Ediger et al., but can be useful in the analysis of the crystal growth rate of some systems,

$$
U_{\mathrm{kin}}^{2 \mathrm{D}}=U \exp \left(\frac{Z}{T \Delta G}\right)=C \frac{D}{\lambda^{2}}=C \frac{k_{B} T}{\lambda^{3} \eta},
$$

where $\exp (-Z / T \Delta G)$ is related to the thermodynamic barrier, in the same form as the denominator in Eq. (9a).

Ediger et $a l .{ }^{9}$ noted that for the organic material tris- $\alpha$-naphthylbenzene ${ }^{12}$ at temperatures below the maximum, the growth rate is dominated by $U_{\text {kin }}$ and supposed that this feature is generic for all liquids that are capable of being substantially undercooled. The present authors verified the validity of this assumption for several silicates, borates, and for three other organic materials. ${ }^{13}$ As Ediger et al. ${ }^{9}$ focused on $U_{\text {kin }}$, they restricted their analysis to liquids for which crystal growth rates have been measured at temperatures below the growth rate maximum and considered only Eq. (9a) for all systems. Below, we further extend the analysis of Ref. 9.

\section{VISCOUS FLOW AND FRAGILITY}

The viscosities of most silicate melts are well described by the empirical equation of Vogel-Fulcher-TammannHesse (VFTH), ${ }^{14-16}$

$$
\log _{10} \eta=A+\frac{B}{T-T_{0}},
$$

where $A, B$, and $T_{0}$ (the Kauzmann temperature) are empirical parameters.

The VFTH expression was independently proposed by several authors: Vogel ${ }^{14}$ developed this equation in 1921 based on the investigations of the temperature dependence of the viscosity of some simple liquids, such as water, mercury, and oil, but not glass-forming liquids. Fulcher ${ }^{15}$ analyzed the viscosities of several silicate glasses in 1925, and in 1926, Tammann and Hesse ${ }^{16}$ analyzed their experiments with glass-forming organic substances based in this equation. The VFTH equation can also be derived in the framework of both the Adam-Gibbs ${ }^{17}$ and the free volume theories of liquids. ${ }^{18}$

A useful way to understand structural arrangements occurring with temperature is by means of the so-called fragility parameter introduced by Angell, ${ }^{19}$

$$
m=\left.\frac{d\left(\log _{10} \eta\right)}{d\left(T_{g} / T\right)}\right|_{T=T_{g}},
$$

which indicates the variation of viscosity with temperature at $T_{g}$. Glass forming liquids are characterized as strong, moderate, or fragile, depending on the value of $m$. For instance, $m<20$ for strong liquids and can be as high as 100 for fragile oxide glass formers.

Tridimensionally bonded network liquids, such as $\mathrm{SiO}_{2}$ or $\mathrm{GeO}_{2}$, with highly polymerized networks (which are virtually free from nonbridging oxygens) barely change their structure and activation energy for viscous flow with temperature showing an Arrhenius dependence of viscosity between $T_{g}$ and the liquidus. These liquids are classified as "strong" with $m \sim 20$. All the other glass-forming systems are classified as "moderate" and "fragile." For fragile liquids, the viscosities vary in a stoutly non-Arrhenius fashion with a larger value of $m(m>50)$, and their apparent activation energies significantly change with temperature. The extremely fast rise of viscosity is one of the most striking properties of fragile glasses: it can increase by ten orders of magnitude as the temperature is decreased by a factor of 2 .

The viscosity of good glass-forming liquids at the equilibrium melting point or liquidus is typically larger than $\sim 10^{2} \mathrm{~Pa} \mathrm{~s}$, while for poor glass formers, $\eta<10 \mathrm{~Pa} \mathrm{~s}$ at the liquidus, and the viscosity dramatically increases upon cooling, reaching $\sim 10^{12} \mathrm{~Pa} \mathrm{~s}$ at the laboratory glass transition $T_{g}$. A decrease in temperature reduces the atomic mobility, and thus some systems can be easily vitrified before crystallization, while others require quite high cooling rates.

The decoupling of effective diffusion and viscosity may lead to modifications of the classical expressions for crystal growth rates and can be one of the reasons for a deviation of theoretical predictions from experimental crystal growth data. $D_{U}$ (Refs. 4 and 5) could be related to the diffusion coefficient of the ambient phase units and to their characteristic size $\lambda$ (or average jumping distance). For instance, diffusivity calculated from viscosity is generally different from self-diffusion of alkalis in ionic conducting glasses. ${ }^{20}$ For fragile systems-such as the multicomponent systems focused in our paper-it is not trivial to determine what these diffusing "units" are. In these cases, Schmelzer ${ }^{1}$ proposed that the actual diffusion coefficient must be a combination of the diffusion coefficients of all components $D_{\text {eff }}$ and that this parameter probably may not have a direct correspondence with that calculated from the Newtonian viscosity.

\section{OBJECTIVES OF THIS RESEARCH}

In this article, we test the interesting proposal of Ediger et $a l .{ }^{9}$ for the $U_{\text {kin }}$ versus $\eta$ relationship by analyzing the crystal growth rates using different growth models plus viscosity and thermodynamic data in a wide temperature range, between $\sim T_{g}$ and $T_{m}$ for strong, moderate, and fragile oxide glass-forming systems. We selected several systems according to the following criteria: (i) the liquid and equilibrium crystal compositions are the same; (ii) the crystal growth is linear in time in isothermal experiments; (iii) crystal growth rates are available in a wide temperature range, from slightly above $T_{g}$ to $T_{m}$ (we include our own data for diopside: $\mathrm{CaO} \cdot \mathrm{MgO} \cdot 2 \mathrm{SiO}_{2}$ and $\mathrm{PbO} \cdot \mathrm{SiO}_{2}$ ). We considered nine glass-forming systems that preferentially crystallize from the sample surface, plus one that also crystallizes in the interior (lithium disilicate, $\mathrm{LS}_{2}$ ). The tested glasses have fragility within the range of $20<m<60$. 

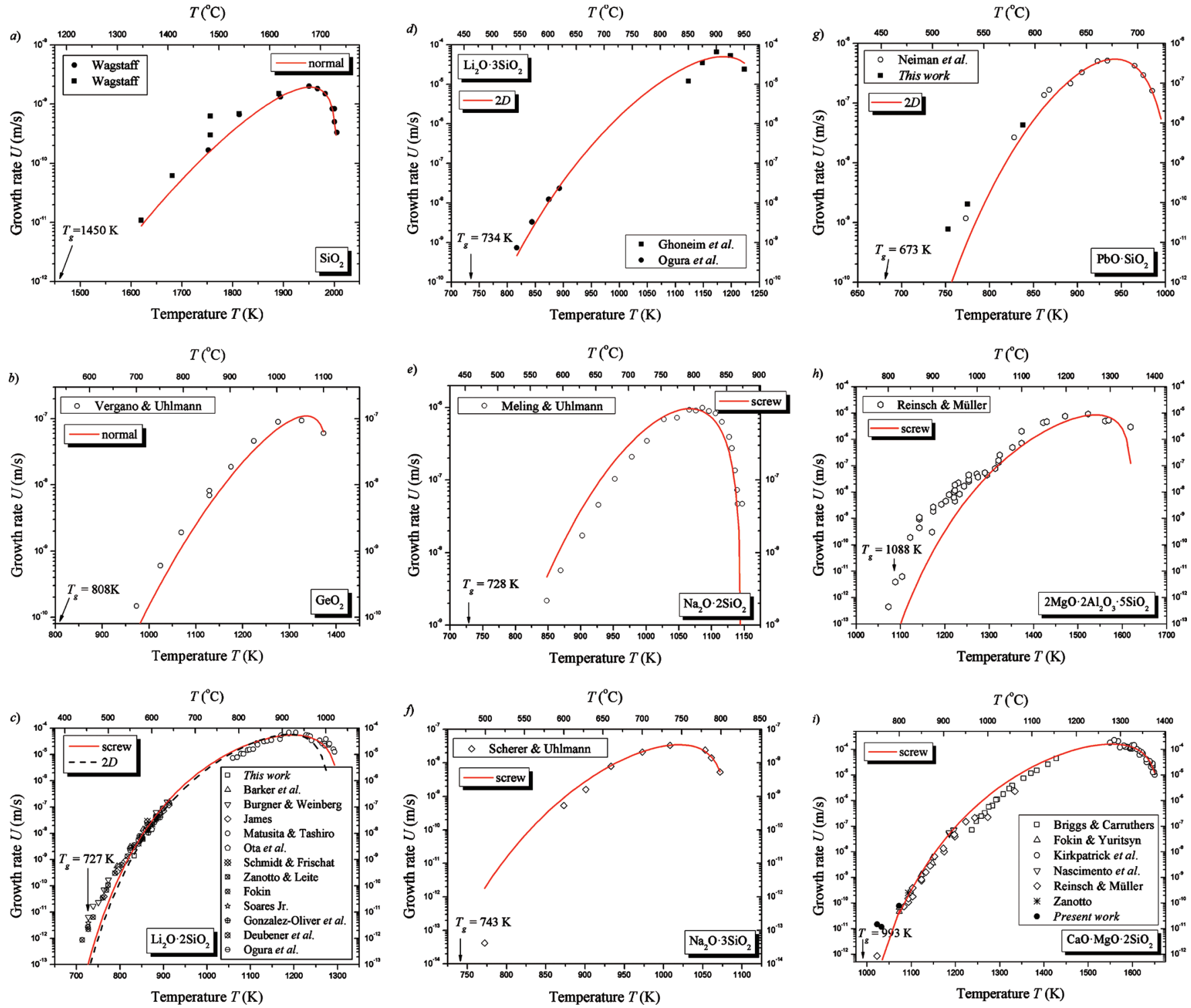

FIG. 2. [(a)-(i)] Experimental crystal growth rates for nine glass-forming liquids. The lines are fitted curves using the crystal growth model that best fits each data set. For $\mathrm{LS}_{2}$ glass, we also show a dashed line corresponding to the use of a second growth mechanism. Viscosity and growth rate data for $\mathrm{SiO}_{2}$, $\mathrm{GeO}_{2}$, $\mathrm{LS}_{2}, \mathrm{LS}_{3}, \mathrm{NS}_{3}, \mathrm{PS}$, diopside, and cordierite (shown in Table I) are averaged values from several authors. However, for these fragile highly depolymerized glasses, there is only a relatively small scatter between data from different sources using different glass melts. A different behavior is shown by the strong, highly polymerized silica glass (Ref. 4), which is extremely sensitive to the impurity content. Special care in analyzing the data was taken in this case (Ref. 4).

From the proposal of Ediger et al. [Eq. (12)], one can plot $U_{\text {kin }}$ versus $\eta$ in the log-log scale for each composition, which in their paper yields straight lines. One can then assess a power law dependence of $U_{\text {kin }}$ on the liquid viscosity, with a slope $\xi$,

$$
U_{\text {kin }} \propto \eta^{-\xi},
$$

where values of the exponent $\xi<1$ express a decoupling of viscosity from the transport part of the crystal growth in the temperature range of study. In this article, we will go deeper into this question by analyzing in detail the crystal growth and viscosity data in a wider temperature range for nine silicate glasses to confirm the possibility of decoupling.

\section{RESULTS}

Figure 2 shows the experimental crystal growth rate data for nine inorganic glass-forming systems ${ }^{4,5,13,21-28}$ and the classical crystal growth models that best fit the data with only one adjustable parameter, i.e., the jumping distance $\lambda$, for the normal and screw dislocation models. For the 2D model, $\lambda$ was fixed and two fitting parameters were used: $N_{S}$ and $\gamma$. These parameters are largely temperature independent. The respective $T_{g}$ 's are indicated for all systems.

According to Table I, for the SD model the fitted $\lambda$ is between 0.15 and $3 \AA$ for all systems, which are acceptable values for the undefined "structural units," as explained above (all the experimental, model, and fitting errors are embedded in this parameter).

The normal growth model describes well the growth ki- 
TABLE I. VFTH viscosity parameters ( $\eta$ in Pa s and $T$ in K) and fitted crystal growth rate parameters between $T_{g}$ and $T_{m}$. The respective fragility index $m$ for nine selected inorganic glass-forming systems. The crystal growth model used (normal, screw dislocation, or 2D) is indicated. We also compared by means of Eq. (14) the slopes of the curves in Fig. 5 (denominated $\xi^{\prime}$ ), which showed systematically higher values than $\xi$ calculated using the definition of Ediger $e t$ al. [Eq. (12)]. For the calculation of $\xi$ and $\xi^{\prime}$, the growth rate data from near the melting point down to temperatures as low as $0.98 T_{g}$ were used, as shown above, where the lowest temperatures for which crystal growth rate data are available. Notes: for cordierite, the crystal growth mechanism is uncertain because both SD and 2D can be used due to the uncertainty in the $T_{m}$ of $\mu$-cordierite. Using $T_{m}$ as $1623 \mathrm{~K}$, the best fit is obtained with the screw dislocation model. For $\mathrm{LS}_{2}$, both SD and $2 \mathrm{D}$ growth mechanisms could be used, but the former gives the best fit.

\begin{tabular}{|c|c|c|c|c|c|c|c|c|c|c|c|c|c|}
\hline Glass & Model & $A$ & $\begin{array}{c}B \\
(\mathrm{~K})\end{array}$ & $\begin{array}{l}T_{0} \\
(\mathrm{~K})\end{array}$ & $m$ & $\begin{array}{l}\lambda \\
(\AA)\end{array}$ & $\begin{array}{c}N_{S} \\
\left(\mathrm{~m}^{-2}\right)\end{array}$ & $\begin{array}{c}\gamma \\
\left(\mathrm{J} / \mathrm{m}^{2}\right)\end{array}$ & $\xi$ [Eq. (12)] & $\xi^{\prime}$ [Eq. (14)] & $\begin{array}{c}V_{m} \\
\left(10^{-5} \mathrm{~m}^{3} / \mathrm{mol}\right)\end{array}$ & $\begin{array}{c}\Delta H_{m} \\
(\mathrm{~kJ} / \mathrm{mol})\end{array}$ & $\begin{array}{l}T_{c} / T_{g} \\
(\mathrm{~K} / \mathrm{K})\end{array}$ \\
\hline $\mathrm{SiO}_{2}$ & $N$ & -8.811 & 30193.8 & 0 & 21 & 1.99 & $\cdots$ & $\cdots$ & $0.95 \pm 0.03$ & $0.94 \pm 0.03$ & 2.37 & 7.68 & 1.12 \\
\hline $\mathrm{GeO}_{2}$ & $N$ & -5.997 & 14535.8 & 0 & 22 & 3.06 & $\cdots$ & $\cdots$ & $0.95 \pm 0.01$ & $0.94 \pm 0.02$ & 2.46 & 12.98 & 1.20 \\
\hline $\mathrm{Li}_{2} \mathrm{O} \cdot 2 \mathrm{SiO}_{2}$ & Screw/2D & -2.623 & 3388.8 & 491.1 & 35 & $0.35 / 1.0$ & $1.48 \times 10^{16}$ & 0.150 & $0.82 \pm 0.01$ & $0.81 \pm 0.01$ & 6.15 & 57.3 & $0.98^{\mathrm{b}}$ \\
\hline $\mathrm{Li}_{2} \mathrm{O} \cdot 3 \mathrm{SiO}_{2}$ & $2 \mathrm{D}$ & -4.668 & 7732.0 & 274.6 & 25 & 1.0 & $5.57 \times 10^{21}$ & 0.178 & $0.92 \pm 0.02$ & $0.90 \pm 0.01$ & $6.15^{\mathrm{a}}$ & $57.3^{\mathrm{a}}$ & 1.12 \\
\hline $\mathrm{Na}_{2} \mathrm{O} \cdot 2 \mathrm{SiO}_{2}$ & Screw & -3.075 & 4595.8 & 392.9 & 36 & 0.15 & $\cdots$ & $\cdots$ & $0.94 \pm 0.01$ & $0.93 \pm 0.14$ & 7.09 & 33.45 & 1.16 \\
\hline $\mathrm{Na}_{2} \mathrm{O} \cdot 3 \mathrm{SiO}_{2}$ & Screw & -2.687 & 4451.5 & 427.5 & 27 & 1.12 & $\cdots$ & $\ldots$ & $0.93 \pm 0.05$ & $0.91 \pm 0.05$ & 7.35 & 36.05 & 1.17 \\
\hline $\mathrm{PbO} \cdot \mathrm{SiO}_{2}$ & $2 \mathrm{D}$ & -4.390 & 3188.1 & 481.5 & 40 & 1.0 & $2.62 \times 10^{13}$ & 0.162 & $0.83 \pm 0.02$ & $0.77 \pm 0.02$ & 4.496 & 33.89 & $1.11^{\mathrm{b}}$ \\
\hline $2 \mathrm{MgO} \cdot 2 \mathrm{Al}_{2} \mathrm{O}_{3} \cdot 5 \mathrm{SiO}_{2}$ & Screw & -3.97 & 5316.0 & 762.0 & 50 & 1.19 & $\ldots$ & $\cdots$ & $0.74 \pm 0.02$ & $0.73 \pm 0.02$ & 22.59 & 175.0 & $0.98^{\mathrm{b}}$ \\
\hline $\mathrm{CaO} \cdot \mathrm{MgO} \cdot 2 \mathrm{SiO}_{2}$ & Screw & -4.680 & 4463.6 & 726.8 & 59 & 3.69 & $\ldots$ & $\ldots$ & $0.85 \pm 0.01$ & $0.84 \pm 0.04$ & 7.59 & 138.0 & $1.03^{\mathrm{b}}$ \\
\hline
\end{tabular}

${ }^{\mathrm{a} E s t i m a t e d ~ v a l u e s ~ f r o m ~ l i t h i u m ~ d i s i l i c a t e ~ g l a s s . ~}$

${ }^{\mathrm{b}}$ Fragile glasses that show a kink at $T / T_{g} \sim 1.2-1.1$ in the kinetic coefficient versus viscosity plot.

${ }^{\mathrm{c}} T_{c}$ is the lowest temperature for which crystal growth rate data is available.

netics for silica $\left(\mathrm{SiO}_{2}\right)[\mathrm{Fig} .2(\mathrm{a})]$ and germania $\left(\mathrm{GeO}_{2}\right)[\mathrm{Fig}$. 2(b)]. It is important to note that we also tested the other classical models and found that they do not apply for these glasses.

Regarding the 2D growth mechanism, we fixed one of the parameters (the jumping distance, $\lambda=1 \AA$ ) and varied $N_{S}$ and $\gamma$. The resulting values presented in Table I are reasonable. For example, lithium disilicate $\left(\mathrm{LS}_{2}\right)$ is a case for which both SD and 2D models could be equally applicable, but the screw dislocation model provides a slightly better fit [the full line in Fig. 2(c)]. The $N_{S}$ and $\gamma$ values from the 2D model are quite similar to those for two other systems (lead silicate (PS) [Fig. 2(g)] and lithium trisilicate $\left(\mathrm{LS}_{3}\right)$ [Fig. 2(d)]). There is not enough growth rate data for $\mathrm{LS}_{3}$, sodium disilicate $\left(\mathrm{NS}_{2}\right)$, and sodium trisilicate $\mathrm{NS}_{3}$ glasses, but the models used here agree quite well with the available data. For $\mathrm{NS}_{3}$ [Fig. 2(f)], however, the lowest crystal growth rate datum departs somewhat from the predicted curve.

We also show our own experimental data at low temperatures for diopside glass. The crystal growth data for diopside at low temperatures correspond to observable single crystals, as well as to layer crystallization. ${ }^{13}$ A kink occurs near $750{ }^{\circ} \mathrm{C}$ [Fig. 2(i)]; our experimental data below and above Reinsch and Müller's data show somewhat faster growth kinetics (possibly, Reinsch and Müller's datum at $750{ }^{\circ} \mathrm{C}$ is only approximate due to the experimental difficulty in obtaining $U$ at such low temperatures, which require very long heat treatments-i.e., several weeks).

Regarding the cordierite glass $\left(2 \mathrm{MgO} \cdot 2 \mathrm{Al}_{2} \mathrm{O}_{3} \cdot 5 \mathrm{SiO}_{2}\right)$, the melting point of (metastable) $\mu$-cordierite crystals cannot be directly measured. Therefore, we assume that its upper bound is the melting point of the stable high temperature polymorph of cordierite (indialite, $h$ - or $\alpha$-cordierite) at $1467{ }^{\circ} \mathrm{C}$. The lower bound of $T_{m}$ is assumed to be $\approx 1350{ }^{\circ} \mathrm{C}$ since metastable $\mu$-cordierite is detectable as the primary crystal phase up to $1300{ }^{\circ} \mathrm{C}$ and is best fitted with the 2D growth model equation. For higher $T_{m}$, the best choice is obtained applying the screw dislocation mechanism.

However, growth rates in cordierite glass can be described by the so-called "combined model.", ${ }^{27}$ Thus, the experimental data (including the kinks) could be fitted for the whole temperature range by a linear combination of two growth models. Such fittings indicate that viscosity can be used to assess the transport mechanism that determines crystal growth in this system, from the melting point $T_{m}$ down to $T_{g}$, with no breakdown of the Stokes-Einstein/Eyring equation.

In all plots shown below, one or another model describes fairly well, but not perfectly, the growth rate versus temperature curves, with some departure from the experimental data at the lowest temperatures. These results corroborate the validity of the SE/E expression at high temperatures (average and low viscosities) and point to some breakdown at lower temperatures. These features will be tested in the following sections of this article.

Table I shows the respective VFTH viscosity parameters, considering the same temperature range used for the analysis of crystal growth data, as well as the fragility index $m$. This parameter confirms that silica is the strongest and diopside is the most fragile among the nine systems. For these silicates, the fragility index corresponds to the depolymerization degree or to the increase of the alkali content-as noted for sodium and lithium di- and trisilicates. Lithium, sodium, and potassium disilicates have about the same $m$, while the metasilicates (lead metasilicate, diopside, and cordierite) are the most fragile, as expected. Table I also shows the $\xi$ parameter calculated from all the experimental crystal growth rates using the original $U_{\text {kin }}$ of Ediger et al..$^{9}$ [Eq. (12)]. Lithium disilicate, lead metasilicate, cordierite, and diopside, for which crystal growth rate data are available at the lowest temperatures, $T<1.1 T_{g}$, show the lowest $\xi$. This result indicates that if extensive data were available at lower tempera- 


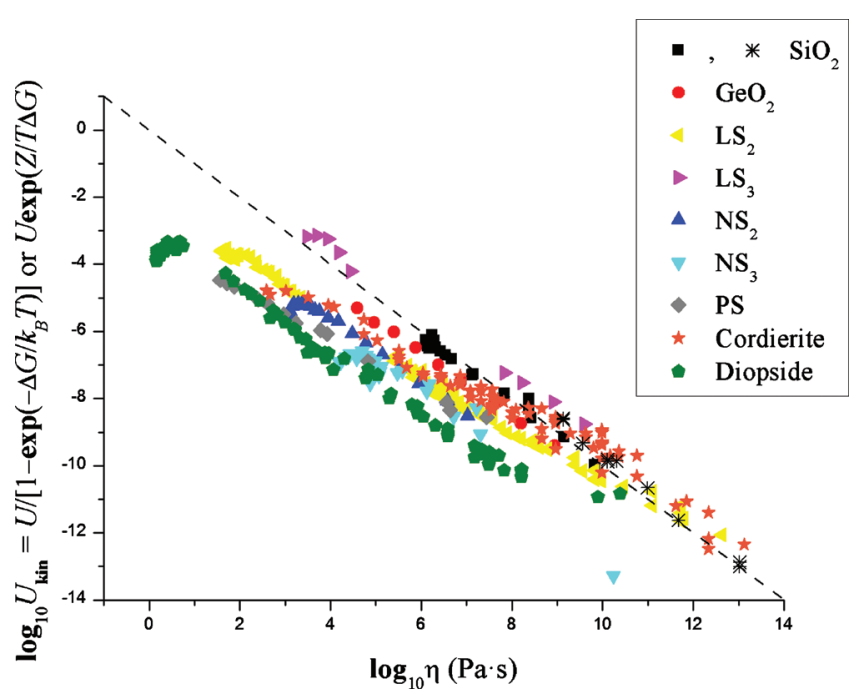

FIG. 3. Original kinetic coefficient $\left(U_{\text {kin }}\right)$ proposed by Ediger et al. (Ref. 9) using the normal growth model. There is a deviation from the -1 slope and of the vertical positions from the expected $\eta^{-1}$ (dashed line) for most glasses (fragile), except for the two strong glasses $\mathrm{SiO}_{2}$ and $\mathrm{GeO}_{2}$. For silica glass (*) means the silicon diffusion data from Brebec et al. and (ם) refers to the measured crystal growth rate. For more details, please check Refs. 5 and 13.

tures for other systems perhaps the values of $\xi$ would be modified. Silica and germania are well-known strong glasses, for which crystal growth can be described by the normal growth mechanism, and $\xi$ is indeed close to unity. Below, we discuss the sensitivity of $\xi$ to different ways of calculating it.

\section{DISCUSSION}

Figure 3 shows the $U_{\text {kin }}$ versus viscosity dependence in the $\log -\log$ scale for the nine systems in the previous figure. It is important to note that the extreme points (corresponding to the lowest viscosities) were obtained at temperatures just below the melting point $T_{m}$. Using the original proposal of Ediger et al. [Eqs. (9a) and (9b)], the plot of $U_{\text {kin }}$ versus $\eta$ shows a departure from the expected straight line of slope (Fig. 3). According to Ediger et al., ${ }^{9}$ the slope $\xi$ scales with the glass fragility. In addition, at high temperatures, near the maximum of the crystal growth rate, a departure from the theoretical line is observable for diopside, cordierite, and $\mathrm{Li}_{2} \mathrm{O} \cdot 2 \mathrm{SiO}_{2}$. We will show below that part of these departures is due to factors that were not used in the original proposal, such as the pre-exponential factors, $f$ and $C / \lambda$, for the screw dislocation and 2D mechanisms, respectively. As $f \sim 1$ for the normal growth model, the original $U_{\text {kin }}$ scales with $\eta^{-1}$ for silica and germania.

Figure 3 shows that for silica glass the directly measured silicon diffusivity is the same as that calculated from crystal growth rate and from viscosity (for viscosities up to $\left.10^{13} \mathrm{~Pa} \mathrm{~s}\right): U_{\mathrm{kin}}=D / \lambda=k_{B} T / \lambda^{2} \eta$, as previously observed. ${ }^{5,13}$ This means that for $\mathrm{SiO}_{2}$ the $\mathrm{SE} / \mathrm{E}$ equation works from the melting point down to $\sim T_{g}$.

In this article, we modify Eqs. (9a) and (9b) by inserting temperature dependent parameters $f(T)$ from Eq. (2) and $C(T)$ from Eq. (7), giving Eqs. (13a) and (13b),

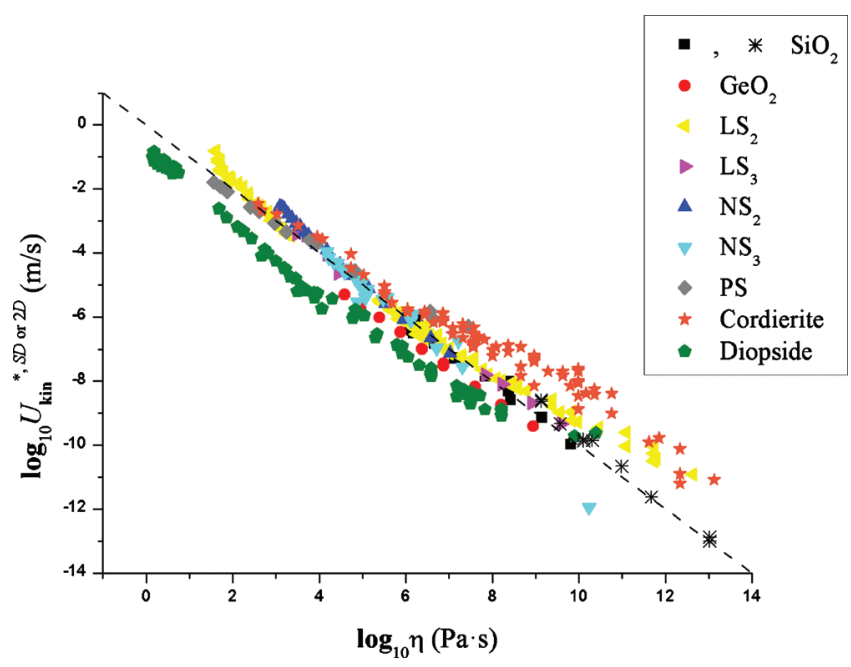

FIG. 4. Modified kinetic coefficient $\left(U_{\text {kin }} / f\right)$ or $\left(U_{\text {kin }} \lambda / C\right)$, where $f$ is the pre-exponential interface site factor $\left(f \approx \Delta T / 2 \pi T_{m}\right)$ from Eq. (2) or $C / \lambda$ from Eqs. (7a) and (7b), respectively. A deviation from the expected $\eta^{-1}$ behavior (dashed line) is still observed for some fragile glasses.

$$
U_{\mathrm{kin}}^{* N, \mathrm{SD}}=\frac{U}{f\left[1-\exp \left(-\Delta G / k_{B} T\right)\right]}=\frac{k_{B} T}{\lambda^{2} \eta}
$$

and

$$
U_{\text {kin }}^{* 2 \mathrm{D}}=\frac{U \lambda}{C} \exp \left(\frac{Z}{T \Delta G}\right)=\frac{k_{B} T}{\lambda^{2} \eta} .
$$

For silica and germania, which display normal growth, the application of Eq. (13a) does not modify the results of Ediger et al. ${ }^{9}$ because $f$ is a constant between 0.5 and 1 for this growth mechanism.

However, the use of Eqs. (13a) and (13b) in Fig. 4 confirms that the factor $f(T)$ for screw dislocation growth-in the form $U_{\text {kin }} / f$-or the factor $U_{\text {kin }} \lambda / C$ for $2 \mathrm{D}$ growth eliminates most of the discrepancy observed at high temperatures and approaches the experimental data to the expected viscosity curve (dashed line, $\eta^{-1}$ ). The new $U_{\text {kin }}^{*}$ from Eqs. (13a) and (13b) are similar to the previous one given by Eqs. (9a) and (9b), but now the product of crystal growth and viscosity depends on the temperature and jump distance $\lambda$. This explains the departure from the dashed line in Fig. 4 for some systems.

Perhaps, a better way to compare the kinetic part of crystal growth with viscosity is by comparing parameters having the same units (i.e., by defining a normalized kinetic term $M_{\text {kin }}$ in $1 / \mathrm{Pa} \mathrm{s}$ ). Thus, we propose the following equations:

$$
M_{\text {kin }}^{\mathrm{SD}}=\frac{U \lambda^{2}}{f k_{B} T\left[1-\exp \left(-\Delta G / k_{B} T\right)\right]}
$$

and

$$
M_{\mathrm{kin}}^{2 \mathrm{D}}=\frac{U \lambda^{3}}{C k_{B} T} \exp \left(\frac{Z}{T \Delta G}\right) .
$$

Figure 5 shows that by considering the term $U_{\text {kin }} \lambda^{2} / f k_{B} T$ for the screw dislocation and 2D growth models, the deviations between the calculated $M_{\text {kin }}$ and the measured viscosity 


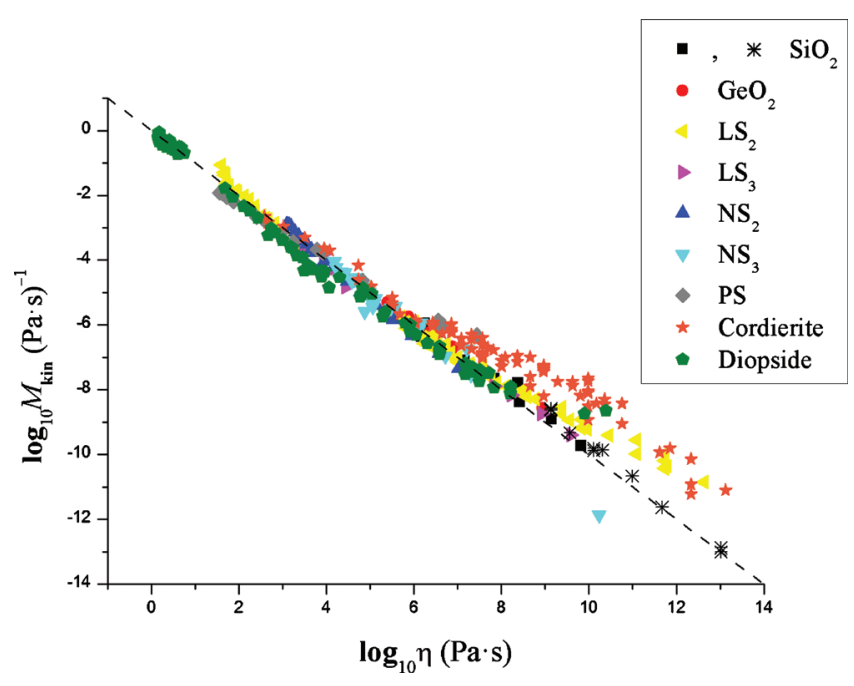

FIG. 5. Normalized kinetic coefficients $\left(U_{\mathrm{kin}} \lambda^{2} / f k_{B} T\right)$ or $\left(U_{\mathrm{kin}} \lambda^{3} / C k_{B} T\right)$, where $f$ is the pre-exponential interface site factor $\left(f \approx \Delta T / 2 \pi T_{m}\right)$ from Eq (2) or $C / \lambda$ from Eqs. (7a) and (7b), respectively, and $\lambda$ is the jump distance.

almost disappear at high temperatures-low viscosities. At high temperatures, all systems follow the SE/E equation, as previously shown in Figs. 2(a)-2(i).

However, at low temperatures (viscosities $>10^{6} \mathrm{~Pa} \mathrm{~s}$ ), there is a departure of $\eta^{-1}$ for four fragile systems: cordierite, diopside, lead metasilicate, and lithium disilicate. Such departures are noted because crystal growth data are available near $T_{g}$ for these four systems, and some are presented here for first time. For the five other systems analyzed in this paper, the growth rate data are only available at somewhat higher temperatures above $1.1 T_{g}-1.2 T_{g}$.

Just for comparison, we calculated another slope parameter, $\xi^{\prime}$, from Fig. 5, following the definition of Ediger et al., ${ }^{9}$ but based on Eqs. (14a) and (14b), and shown in Fig. 6. We used the data available for the whole temperature range in the fitting procedure to obtain the values of $\xi^{\prime}$. The results shown in Table I are lower than the original $\xi$ of Ediger et $a l .{ }^{9}$ For the strong systems such as silica and germania, the slope is very close to -1 . The calculated $\xi^{\prime}$ parameters are

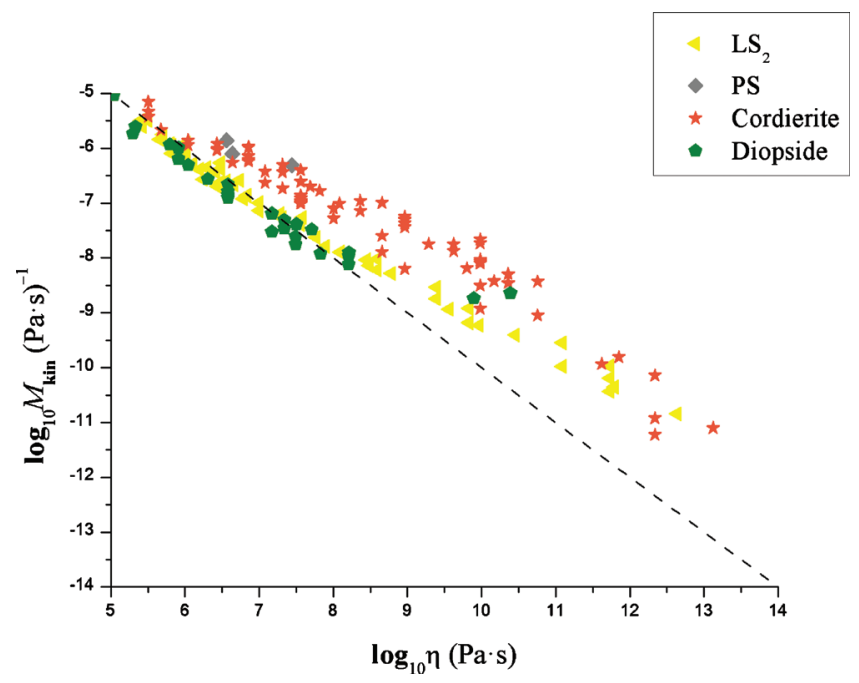

FIG. 6. Details of the previous figure showing only the four fragile silicate systems that present a kink at $T_{d}$. lower than 0.95 for five glasses, but are significantly smaller for four systems ( $\mathrm{LS}_{2}, \mathrm{PS}$, diopside, and cordierite), which will be discussed below. For the calculation of $\xi$ and $\xi^{\prime}$, the growth rate data from just below the melting point down to temperatures as low as $0.98 T_{g}$ were used (please check Table I for more details).

\section{A. Signs of a breakdown of the SE/E equation for four fragile glasses}

Let us now focus on the four systems that show signs of decoupling at $T_{d}$ between viscous flow and the transport part controlling the crystal growth. As regards the $\mu$-cordierite [Fig. 2(h)], there is a noticeable shoulder at $\sim 1.2 T_{g}$, exactly where a possible breakdown of the SE/E equation could be expected. However, for real decoupling between viscous flow and effective diffusivity - such as those reported in Refs. 6-8 for organic materials and in Ref. 29 for metallic glasses-below $\sim 1.2 T_{g}$, the Stokes-Einstein/Eyring equation would no longer describe the diffusion coefficients and a permanent change in the slope, not only a kink in the curve, would be observed. However, in Ref. 27, a fitting of $U(T)$ including this shoulder in Fig. 2(h) was performed, assuming that the melt/crystal interface, growing from screw dislocation defects, is additionally roughened by superimposed 2D surface nucleation at large undercoolings below $1.2 T_{g}$. Thus, this supposition of a "combined" growth mechanism might be feasible, although no clear evidence can be claimed. However, other possibilities can be considered to account for this phenomenon, which may also affect the assessed growth mechanism and the fitted parameters, for instance, compositional changes within the crystal (such as those reported in Refs. 30 and 31), and changes of the crystal phase and morphology.

Small effects, such as the observed shoulder in $U(T)$ for cordierite, might be caused by changes of the chemical composition of the growing crystals with annealing time or temperature. Such changes in silicates have been experimentally verified in other silicate glasses by Fokin et al. ${ }^{30}$ and Roskosz et al., ${ }^{31}$ but not in all glasses. For instance, considering diopside, preliminary results of quantitative EDS analysis by SEM (crystallized layer heat treated at $890{ }^{\circ} \mathrm{C}$ for $16 \mathrm{~h}$ ) showed no difference between the glassy and the crystal phase compositions. In addition, to the best of our knowledge, no chemical changes have ever been reported for the crystal phases of the other two systems of interest here: $\mathrm{LS}_{2}$ and PS.

Possible morphology changes in diopside, $\mathrm{LS}_{2}$, and PS crystals growing in their isochemical glass have not been exhaustibly tested, but, to the best of our knowledge, there is no published evidence for such changes. We should note, however, that for $\mu$-cordierite, Diaz-Mora ${ }^{32}$ observed a change in morphology from elongated hexagonal crystals to regular hexagons at $T<900{ }^{\circ} \mathrm{C}$. Small crystals $<2 \mu \mathrm{m}$, grown at $T<900{ }^{\circ} \mathrm{C}$, were observed at the melt/crystal interfaces, whereas large crystals grown at $T>1000{ }^{\circ} \mathrm{C}$ are dendritically structured. Nevertheless, the shape of the isolated crystals does not deviate from hexagonal bipyramids and their growth rate does not explicitly depend on time (or 
crystal size). Hence, we believe that the influence of morphology may be neglected for the measured crystal growth rate data of $\mu$-cordierite. In summary, for cordierite, a change in the growth mechanism could perhaps explain the observed kink.

However, for the other three systems- $\mathrm{LS}_{2}$, diopside, and PS-below $T_{d}$, the temperature dependences of the kinetic coefficient for growth and viscosity differ. Therefore, there is indeed a possible breakdown of the SE/E equation for these three "suspect" systems. Figure 6 shows clear signs of a departure from the line of slope -1.0 at $T_{d}$ between $1.10 T_{g}$ and $1.15 T_{g}$, equivalent to viscosities of $10^{8}-4$ $\times 10^{6} \mathrm{~Pa} \mathrm{~s}$, respectively. Following the suggestion of one reviewer, another exponent parameter $\left(\xi^{*}\right)$, different from $\xi^{\prime}$, can be obtained from Fig. 6. $\xi^{*}$ was calculated using the low temperature/high viscosity data of the three glasses for which it is possible to note a clear deviation from the StokesEinstein behavior at $T_{d}$. At this range of high viscosities, the slope significantly deviates from the expected -1 curve. For $T<T_{d}, \xi^{*} \approx 0.73 \pm 0.01$ for $\mathrm{LS}_{2}$ and $\xi^{*} \approx 0.70 \pm 0.04$ for diopside. These slopes are equal within the error limits. For lead metasilicate, there are only few data points and the estimated slope $\xi^{*} \approx 0.41 \pm 0.21$ is probably too low, with high uncertainty. These small slopes suggest a decoupling of viscosity and the effective diffusivity controlling crystal growth at such high viscosities.

However, in the present analysis, the success or failure of the SE/E relation depends on the validity of the models of the crystal growth employed. Thus, the venerable SE/E relation is put on trial using arguments based on theories of the crystal growth. But have these theories been similarly tested? Yes, they have been and work quite well for oxide glassforming systems at high temperatures (see, e.g., Refs 4, 5, and 10). However, one might also argue that a change of the crystal growth mechanism could be occurring at $T_{d}$, which could lead to a kink in the curves. However, if this was the case, viscosity would still describe the temperature dependence of the effective diffusivity that controls crystal growth at temperatures below the kink (as observed for cordierite using a combined growth model).

In resume, the present tests cannot clearly distinguish a breakdown of the SE/E equation from a possible change of growth mechanism. However, the first is more likely to occur because a simple change of growth mechanism would produce a kink in the curve, but the viscosity would still describe the temperature dependence of the transport processes controlling crystal growth. In any case, viscosity (via the $\mathrm{SE} / \mathrm{E}$ equation) cannot be used to describe the transport part of crystal growth in fragile liquids in the neighborhood of $T_{g}$. This finding is consistent with the results of Ediger et $a l .{ }^{9}$-our results thus show clear deviations of $M_{\mathrm{kin}}$ from $1 / \eta$ for fragile glasses-and the normalized kinetic coefficient here proposed $\left(M_{\text {kin }}\right)$ is thus an appropriate tool to assess liquid dynamics from crystal growth rate data.

\section{SUMMARY AND CONCLUSIONS}

We revisited the proposal of Ediger et al. that the diffusivities controlling crystal growth rates in some inorganic and organic liquids decouple from inverse viscosity in a manner that systematically depends on the liquid fragility. We proposed a normalized kinetic term and tested it with nine oxide glass-forming systems of different fragilities in a very wide range of viscosities, from $10^{1}$ to $10^{13} \mathrm{~Pa} \mathrm{~s}$.

Our analysis corroborated the ability of the viscosity to describe the transport process controlling crystal growth rates from low to average viscosities $\left(\eta<10^{6} \mathrm{~Pa} \mathrm{~s}\right)$ for all glasses. In addition, for strong glasses, the SE/E equation works well for all viscosities from the melting point down to $T_{g}$ ! Regarding the four fragile liquids for which very low temperature crystal growth data are available down to $<1.1 T_{g}$, for cordierite glass, the kink could, in principle, be explained by a change of the growth mechanism because the temperature dependences of the two diffusion processes are the about same for temperatures above and below the kink. However, in agreement with the findings of Ediger et al., at higher viscosities, there are clear signs of a decoupling of these two kinetic properties for the three other fragile liquids (diopside, lead metasilicate, and lithium disilicate). Thus, we argue that the diffusion coefficients calculated from crystal growth rates and from viscosity via the SE/E equation have the same temperature dependence at relatively low or moderate viscosities, but for fragile liquids, they decouple at some temperature where the viscosity is high enough. Hence, in general, viscosity cannot be used to analyze or predict crystal growth kinetics of fragile glasses in the high viscosity range.

\section{ACKNOWLEDGMENTS}

We are thankful to Brazilian funding agencies CNPq and FAPESP for Grant Nos. 305373/2009-9, 04/10703-0, and 07/ 08179-9. We are also indebted to Professor Mark D. Ediger, Professor Lian Yu, Professor Jürn W. P. Schmelzer, and Professor Vladimir M. Fokin for their very valuable critical comments.

${ }^{1}$ J. W. P. Schmelzer, J. Non-Cryst. Solids 354, 269 (2008).

${ }^{2}$ H. A. Wilson, Philos. Mag. 50, 238 (1900).

${ }^{3}$ J. Frenkel, Phys. Z. Sowjetunion 1, 498 (1932).

${ }^{4}$ M. L. F. Nascimento, E. B. Ferreira, and E. D. Zanotto, J. Chem. Phys. 121, 8924 (2004).

${ }^{5}$ M. L. F. Nascimento and E. D. Zanotto, Phys. Rev. B 73, 024209 (2006).

${ }^{6}$ S. F. Swallen, P. A. Bonvallet, R. J. McMahon, and M. D. Ediger, Phys. Rev. Lett. 90, 015901 (2003).

${ }^{7}$ S. F. Swallen, K. Traynor, R. J. McMahon, M. D. Ediger, and T. E. Mates, J. Phys. Chem. B 113, 4600 (2009).

${ }^{8}$ M. K. Mapes, S. F. Swallen, and M. D. Ediger, J. Phys. Chem. B 110, 507 (2006).

${ }^{9}$ M. D. Ediger, P. Harrowell, and L. Yu, J. Chem. Phys. 128, 034709 (2008).

${ }^{10}$ D. R. Uhlmann, Nucleation and Crystallization in Glasses, Advances in Ceramics Vol. 4 (American Ceramic Society, Ohio, 1982).

${ }^{11}$ K. A. Jackson, D. R. Uhlmann, and J. D. Hunt, J. Cryst. Growth 1, 1 (1967).

${ }^{12}$ J. H. Magill and D. J. Plazek, J. Chem. Phys. 46, 3757 (1967).

${ }^{13}$ M. L. F. Nascimento, Ph.D. thesis, Federal University of São Carlos, 2004 (in Portuguese).

${ }^{14}$ H. Vogel, Phys. Z. 22, 645 (1921).

${ }^{15}$ G. S. Fulcher, J. Am. Ceram. Soc. 8, 339 (1925).

${ }^{16}$ G. Tammann and W. Hesse, Z. Anorg. Allg. Chem. 156, 245 (1926).

${ }^{17}$ G. Adam and J. H. Gibbs, J. Chem. Phys. 43, 139 (1965).

${ }^{18}$ M. H. Cohen and D. Turnbull, J. Chem. Phys. 31, 1164 (1959).

${ }^{19}$ C. A. Angell, J. Phys. Chem. Solids 49, 863 (1988). 
${ }^{20}$ J.-L. Souquet, M. L. F. Nascimento, and A. C. M. Rodrigues, J. Chem. Phys. 132, 034704 (2010).

${ }^{21}$ M. L. F. Nascimento and E. D. Zanotto, Phys. Chem. Glasses 48, 201 (2007).

${ }^{22}$ P. J. Vergano and D. R. Uhlmann, Phys. Chem. Glasses 11, 30 (1970).

${ }^{23}$ G. S. Meiling and D. R. Uhlmann, Phys. Chem. Glasses 8, 62 (1967).

${ }^{24}$ G. W. Scherer and D. R. Uhlmann, J. Cryst. Growth 29, 12 (1975).

${ }^{25}$ G. Scherer, D. R. Uhlmann, C. E. Miller, and K. A. Jackson, J. Cryst. Growth 23, 323 (1974).

${ }^{26}$ G. Scherer and D. R. Uhlmann, J. Cryst. Growth 15, 1 (1972).

${ }^{27}$ S. Reinsch, M. L. F. Nascimento, R. Muller, and E. D. Zanotto, J. Non-
Cryst. Solids 354, 5386 (2008).

${ }^{28}$ M. O. Prado, M. L. F. Nascimento, and E. D. Zanotto, J. Non-Cryst. Solids 354, 4589 (2008).

${ }^{29}$ X. P. Tang, U. Geyer, R. Busch, W. L. Johnson, and Y. Wu, Nature (London) 402, 160 (1999).

${ }^{30}$ V. M. Fokin, O. V. Potapov, E. D. Zanotto, F. M. Spiandorello, V. L. Ugolkov, and B. Z. Pevzner, J. Non-Cryst. Solids 331, 240 (2003).

${ }^{31}$ M. Roskosz, M. J. Toplis, P. Besson, and P. Richet, J. Non-Cryst. Solids 351, 1266 (2005)

${ }^{32}$ N. Diaz-Mora, Ph.D. thesis, Federal University of São Carlos, 1994 (in Portuguese). 